\title{
PCNL - A comparative study in nonoperated and in previously operated (open nephrolithotomy/pyelolithotomy) patients - A single-surgeon experience
}

\author{
Rahul Gupta, Arti Gupta, Gursimran Singh, Abhineet Suri, Sandeep K Mohan, C.L.Gupta \\ Department of Surgery Government Medical College, Jammu and Kashmir, India
}

\begin{abstract}
Purpose: Re-procedure in patients with history of open stone surgery is usually challenging due to the alteration in the retroperitoneal anatomy. The aim of this study was to determine the possible impact of open renal surgery on the efficacy and morbidity of subsequent percutaneous nephrolithotomy (PCNL).

Materials and Methods: From March 2009 until September 2010, 120 patients underwent PCNL. Of these, 20 patients were excluded (tubeless or bilateral simultaneous PCNL). Of the remaining 100, 55 primary patients were categorized as Group 1 and the remaining (previous open nephrolithotomy) as Group 2. Standard preoperative evaluation was carried out prior to intervention, Statistical analysis was performed using SPSS v. 11 with the chi-square test, independent samples t-test, and Mann-Whitney U test. A p-value $<0.05$ was taken as statistically significant.

Results: Both groups were similar in demographic profile and stone burden. Attempts to access the PCS was less in Group 1 compared to Group $2(1.2+12$ vs $3+1.3$ respectively) and this was statistically significant $(p<0.04)$. However, the mean operative time between the two groups was not statistically significant $(\mathrm{p}=0.44)$. Blood transfusion rate was comparable in the two groups $(\mathrm{p}=0.24)$. One patient in Group 2 developed hemothorax following a supra-11 th puncture. Remaining complications were comparable in both groups.

Conclusion: Patients with past history of renal stone surgery may need more attempts to access the pelvicaliceal system and have difficulty in tract dilation secondary to retroperitoneal scarring. But overall morbidity and efficacy is same in both groups.
\end{abstract}

Key words: calculi; kidney; outcomes

Int Braz J Urol. 2011; 37: 739-744

\section{INTRODUCTION}

The prevalence of urolithiasis is approximately 2 to 3 percent in the general population, and the estimated lifetime risk of developing a kidney stone is about $12 \%$ (1). Over time, renal stone management has undergone a dramatic change, beginning from the era of open pyelolithotomy, to the first percutaneous lithotomy (PCNL) in 1976 (2,3). At present PCNL has become the procedure of choice for managing large renal calculi (4).

However, some authors have reported higher rate of treatment failures in those with past history of lumbotomy and others have implicated any preceding renal surgery as a confounding factor affecting the overall outcome $(5,6)$.

Stone recurrence rate is up to $50 \%$ within 5 to 7 years (7), thus increasing the need for re-intervention. Re-operation following open renal surgery would be difficult due to the distortion of the pelvicaliceal anatomy and expected retroperitoneal scarring. The aim of our study was to evaluate the outcome of PCNL in patients with past history of open nephrolithotomy in comparison to those with primary cases. 


\section{MATERIALS AND METHODS}

We reviewed the records of 120 PCNLs performed in our unit between March 2009 and September 2010. Of these, 20 patients who had undergone tubeless or bilateral simultaneous PCNL were excluded from the study. Of the remaining 100, 55 were primary patients were categorized as Group 1 , and the remaining who had history of open nephrolithotomy/pyelolithotomy were categorized as Group 2. Table-1 compares the demographic profile of the two groups. in the ipsilateral pelvicaliceal system (PCS) under fluoroscopy in lithotomy position. The patient was then placed in the prone position for percutaneous access. Transpapillary puncture was made preferably away from the previous incision site, using a three part needle (Angiomed 1.3mm (17.5G)) under fluoroscopy control after retrograde opacification of the pelvicaliceal system via ureteral catheter. An angle tip terumo wire (Radifocus; Terumo wire) was then positioned in the upper ureter. The tract was then dilated initially using serial Teflon dilators up to $10 \mathrm{Fr}$, followed by placement of Alken's rod. The subse-

Table 1 - Demographic profile of patients in both the groups.

\begin{tabular}{lccc}
\hline Parameters & $\begin{array}{c}\text { Group 1 } \\
(\mathbf{n}=\mathbf{5 5})\end{array}$ & $\begin{array}{c}\text { Group 2 } \\
(\mathbf{n}=\mathbf{4 5})\end{array}$ & P VALUE \\
\hline Mean Age(years) & $35.51 \pm 11.1$ & $40.64 \pm 12.3$ & 0.87 \\
Males & $65 \%$ & $60 \%$ & - \\
BMI $\left(\mathrm{kg} / \mathrm{m}^{2}\right)$ & $21.1 \pm 24$ & $22.4 \pm 25$ & 0.65 \\
Stone size(cm) & $2.8 \pm 1.5$ & $3.0 \pm 2.3$ & 0.34 \\
Stone Side(R:L) & $0.8: 1$ & $1: 1.25$ & 0.39 \\
Location Of Stone Calyceal & $15(27.27 \%)$ & $13(28.89 \%)$ & \\
Pyelocalyceal & $25(45.54 \%)$ & $20(44.44 \%)$ & 0.76 \\
Pelvic & $15(33.33 \%)$ & $12(26.67 \%)$ & \\
\hline
\end{tabular}

The indications for PCNL included a stone burden of greater than $1.5 \mathrm{~cm}$ in length and failure of SWL treatment. The stone burden was measured as the product of the two dimensions on plain radiographs. All patients were evaluated with renal function test, hemogram, coagulation profile, urine routine, urine culture sensitivity and ultrasonography. An intravenous urography (IVU) was carried out in all to assess function and plan the puncture. Urinary tract infections detected preoperatively were treated according to antibiotic sensitivity.

\section{Technique}

All PCNLs were performed by the same surgeon. The standard technique followed for the procedure is explained in brief. Following anesthesia, a retrograde catheter ( $5 \mathrm{fr}$ ) was placed cystoscopically quent dilation was achieved using serial Alken metal dilators (usually up to $26 \mathrm{Fr}$ and occasionally $30 \mathrm{Fr}$ depending on the pelvicaliceal dilation and stone burden) and an Amplatz sheath (Cook Surgical) of adequate caliber placed (28 Fr/32 Fr depending on dilation). The PCNL was then completed using Wolf nephroscope (24 Fr) and pneumatic lithotripsy. The fragmented calculi were removed using forceps or suction. On the table, complete clearance was ensured on fluoroscopy and direct nephroscopy. An adequate size nephrostomy was placed at the end of the procedure. Nephrostomy was removed on the second postoperative day after the check x-ray KUB. The nephrostomy tract site was the dressed with sterile dressing. Patient was then discharged with the instructions to remove the dressing after 72 hours and follow-up after one month if asymptomatic. 


\section{Data Analysis}

Stone size $(\mathrm{cm})$, attempts to access PCS, operating time in minutes, intraoperative and postoperative complications, stone-free rate, blood transfusions, duration of hospital stay were examined. Statistical analysis was performed using SPSS v. 11 with the chi-square test, independent samples t-test, and Mann-Whitney U test. A p-value less than 0.05 was considered statistically significant.

\section{RESULTS}

Table-2 shows the overall outcome in the two groups. Both of the groups were comparable in terms of age, sex, side, BMI and stone burden. Mean operative time for Group 1 was shorter $(78.24$ \pm 17.6 mints) compared to Group 2 (82.09 \pm 28.23 $\mathrm{min})$, but this was not statistically significant. The majority (42/45) of the patients in Group 2 had undergone one surgery in the past, except three (two of which had undergone open surgery twice and one three times). The average time from last open surgery to the present percutaneous procedure was 7 \pm 2 years. Attempts to access the PCS was less in Group 1 compared to Group $2(1.2 \pm 1.2$ vs $3 \pm 1.3$ respectively), and this was statistically significant ( $\mathrm{P}$ $<0.04$ ). We used a no. 21 knife blade for the sharp incision of the fascia and the scar tissue in previ- ously operated patients to facilitate the subsequent dilation. We observed that when the approach in Group 2 was not from the incision site, the dilation was easy compared to access gained from the region of scar tissue. This also reduced the probability of guide wire kinking oraccess failure.

Table-2 also compares the complications within the two groups. Overall intraoperative bleeding was encountered in $6 \%(6 / 100)$. Nine percent $(9 / 100)$ of the patients required blood transfusion. Bleeding responded to conservative measures. None of our patients developed pseudoaneurysms, injury to adjoining organs (Bowel). However, one patient in Group 2 developed hemothorax and required chest drain placement. This patient had undergone PCNL for a recurrent calculus by supracostal approach (supra-11th puncture). Eleven of 100 patients developed postoperative fever and that was attributed to pyelonephritis. These patients were treated conservatively with injectable antibiotics (first-generation cephalosporins and aminoglycoside) until they were afebrile and then switched over to oral therapy (oral quinolone) to complete two weeks of medication. Six of our patients developed a persistent nephrostomy site discharge (4 in Group 1 and 2 in Group 2). These patients were evaluated with local nephrostomy site biopsy and were found to have caseating granuloma. They were treated with the anti-tubercu-

Table 2 - Results.

\begin{tabular}{lccc}
\hline Parameters & $\begin{array}{c}\text { Group 1(\%) } \\
(\mathbf{N = 5 5 )}\end{array}$ & $\begin{array}{c}\text { Group 2(\%) } \\
(\mathbf{N}=\mathbf{4 5})\end{array}$ & p VALUE \\
\hline Mean Operative Time(minutes) & $78.24 \pm 17.616$ & $82.09 \pm 28.236$ & 0.44 \\
Average Drop In Hb(gm\%) & $1.1 \pm 0.495$ & $1.39 \pm 0.590$ & 0.12 \\
Bleeding(Intra-op) & $4(7.2)$ & $2(4.4)$ & 0.22 \\
Blood Transfusion & $5(9.09)$ & $4(8.8)$ & 0.24 \\
Pseudoanurysm & 0 & 0 & ----- \\
Hemothorax & 0 & $1(2.2)$ & 0.31 \\
Renal pelvic injury & $1(1.8)$ & $1(2.2)$ & 0.15 \\
Damage to adjoining organs & 0 & 0 & ----- \\
Post Operative Fever & $5(9.09)$ & $6(13.33)$ & 0.29 \\
Stone Clearance & $91.11 \%$ & $93.33 \%$ & 0.69 \\
Hospital stay & $3.11 \pm 0.532$ & $3.13 \pm 0.548$ & 0.84 \\
\hline
\end{tabular}


lar treatment to which they responded well. Overall stone-free rate is comparable in both groups.

\section{DISCUSSION}

Percutaneous nephrolithotomy was introduced in 1976 but it was not until the 1990s that it became an established and preferred procedure for renal stone management $(3,6)$. In the Indian scenario, though PCNL has established itself well in major cities but in the peripheral regions it is still not freely available. As a result, many patients with renal stones are still being treated conventionally with open surgery. With the recurrence rate for renal stones being high (up to 50\% in 5-7 years (7)), these patients often need re-intervention.

Reports have claimed higher failure rates of PCNL in patients with prior open intervention or lumbotomy $(5,6)$. In those series, PCNL failed in almost one-third of patients with a history of lumbotomy. Similar observations were made by Jones and associates (6) who reported a higher complication rate $(24 \%$ vs. $13.6 \%)$, as well as a lower stonefree rate $(51 \%$ vs. $92 \%)$, in those patients with a previous open nephrolithotomy. However, our study did not show any difference in the outcome of PCNL in such patients.

PCNL in the previously-operated patients may be hampered at various stages. At the outset (i.e at the time of puncture and dilation) perirenal fibrosis and retroperitoneal scarring, may cause a problem (8-10). This was depicted in our study too, as we had to make more attempts to access the PCS in previously-operated patients compared to primary cases. A similar observation was made by Margel et al. (8), who required $2.3 \pm 1.9$ vs. $1.2 \pm 1.1$ attempts in secondary vs. primary cases, thus stressing the fact that retroperitoneal scarring does hamper access to the kidney. Also, retroperitoneal and perinephric scarring make the dilation of the tract difficult. This may be attributed to the marginally longer operating time in case of previously operated cases despite patients having similar stone burden compared to primary cases. To avoid this problem some authors have even used Collings knife or optic urethrotome for tract dilation. However, this increased morbidity, so it is not used now $(11,12)$. We have modified our technique by generously incising the expected tract site with a no. 21 knife blade. This we do by going parallel to the puncture needle under fluoro control so as not to damage the renal parenchyma. Additionally, puncturing the calyx of interest through the nonoperated site (scar site) makes the dilation easy. At times (very sparingly) we have used the reverse end of the Alken rod to get an initial access to the PCS by guiding it over the prepositioned Terumo wire (specially, in cases of caliceal scarring). Later the rod is reversed and placed normally for further telescopic dilation. Difficulty in tract dilation has been observed by Kurtulus et al. (10) in their study where in successful tract formation with balloon dilation in one step could be obtained in $83 \%$ of primary cases while in patients with history of open surgery it was possible in only $50 \%$. Moreover, in their series one patient required open surgery due to a failure to create tract despite using Amplatz and balloon dilators in tandem. We did not convert any case to open due to failure to create tract. The disadvantage of telescopic metal dilation reported in literature is the high incidence of pelvic perforation, but this is rare in experienced hands (13). In our series we had one patient in each of the two groups who had small pelvic perforations that responded well to conservative treatment. We feel that excessive force used for dilation should always be avoided (this can be facilitated by the incision of the fascia as discussed), and also the dilators should always be advanced gently under fluoroscopy to prevent this problem.

Some have recommended a preoperative CT scan so as to study the relationship between the adjoining viscera to the kidney following open surgery $(8,10)$. However, this view is not shared by others $(14,15)$. We did not do a CT scan in any of our patients. We prefer IVU as the initial imaging study to show the exact anatomy of the renal collecting system, which for us is important in planning the initial access. In addition, this helps to keep the cost of evaluation to minimum. None in our series developed injury to adjoining organs (bowel).

Margel et al. (8), in their study, recommend choosing upper-pole caliceal puncture to avoid the scar tissue coming in the way of the puncture needle. In our opinion, mere presence of scar should not guide upper calyx puncture as it has significant 
morbidity. In our series, we encountered one hemothorax following a supracostal puncture in a previously operated patient. However, we agree with the fact that the previous incision site should be avoided if possible to facilitate puncture and ease dilation.

Retroperitoneal and caliceal scarring may fix the kidney thus reducing its mobility. In these cases intraoperative manipulation of nephroscope may torque the kidney and cause laceration with bleeding $(14,16)$. Also, this hampers access to the intrarenal calyces affecting the overall clearance (8). Though we observed difficulty in the intrarenal manipulation, it that did not have a bearing on the overall clearance rate $(91.1 \%$ vs. $93.33 \%$ respectively) or the overall transfusion rate in the two groups $(9.09 \%$ vs. $8.8 \%$ respectively). The clearance rate and transfusion rate were comparable to those reported in literature $(8,16,17)$.

\section{CONCLUSIONS}

Our single-surgeon experience has proved that PCNL in a patient with a history of open nephrolithotomy is safe and effective. It can be performed with no fear of higher risk of failure, excessive bleeding, or damage to adjoining organs. Difficulty in access and tract dilation can be overcome by preferably selecting the site of puncture away from the previous scar and a generous but controlled incision of the fibrous tract.

\section{CONFLICT OF INTEREST}

None declared.

\section{REFERENCES}

1. Johnson CM, Wilson DM, O'Fallon WM, Malek RS, Kurland LT: Renal stone epidemiology: a 25year study in Rochester, Minnesota. Kidney Int. 1979; 16: 624-31.

2. James EL, Brian RM, Andrew PE: Surgical Management of Upper Urinary Tract Calculi, Chapter 44; In: Campbell-Walsh urology 9th (ed.), Philadelphia, Saunders. 2007; pp. 1431-508.

3. Fernström I, Johansson B: Percutaneous pyelolithotomy. A new extraction technique. Scand J Urol Nephrol. 1976; 10: 257-9.
4. Kim SC, Kuo RL, Lingeman JE: Percutaneous nephrolithotomy: an update. Curr Opin Urol. 2003; 13: 235-41.

5. Viville C: Percutaneous nephrolithotomy. Personal experience in 100 cases. J Urol (Paris). 1987; 93: 253-8.

6. Jones DJ, Russell GL, Kellett MJ, Wickham JE: The changing practice of percutaneous stone surgery. Review of 1000 cases 1981-1988. Br J Urol. 1990; 66: 1-5.

7. Parmar MS: Kidney stones. BMJ. 2004; 328: 14204.

8. Margel D, Lifshitz DA, Kugel V, Dorfmann D, Lask D, Livne PM: Percutaneous nephrolithotomy in patients who previously underwent open nephrolithotomy. J Endourol. 2005; 19: 1161-4.

9. Lojanapiwat B: Previous open nephrolithotomy: does it affect percutaneous nephrolithotomy techniques and outcome? J Endourol. 2006; 20: 17-20.

10. Kurtulus FO, Fazlioglu A, Tandogdu Z, Aydin M, Karaca S, Cek M: Percutaneous nephrolithotomy: primary patients versus patients with history of open renal surgery. J Endourol. 2008; 22: 2671-5.

11. Davis BE, Noble MJ, Mebust WK: Use of the Collings knife electrode for percutaneous access in difficult endourology cases. J Urol. 1991; 145: 257-61; discussion 261-2.

12. Tzai TS, Chang CL, Hwang IS: An alternative approach to tract dilation using optic urethrotome in percutaneous nephrolithotomy. J Urol. 1987; 137 : 1110-2.

13. Lynch W, Doust B, Golovsky D: Percutaneous dilatation of difficult nephrostomy tracts. Br J Urol. 1992; 70: 455-6.

14. Lojanapiwat B: Does previous open nephrolithotomy affect the efficacy and safety of tubeless percutaneous nephrolithotomy? Urol Int. 2010; 85: 42-6.

15. Basiri A, Karrami H, Moghaddam SM, Shadpour P: Percutaneous nephrolithotomy in patients with or without a history of open nephrolithotomy. J Endourol. 2003; 17: 213-6.

16. Tugcu V, Su FE, Kalfazade N, Sahin S, Ozbay B, Tasci AI: Percutaneous nephrolithotomy (PCNL) in patients with previous open stone surgery. Int Urol Nephrol. 2008; 40: 881-4.

17. Gupta NP, Mishra S, Nayyar R, Seth A, Anand A: Comparative analysis of percutaneous nephrolithotomy in patients with and without a history of open stone surgery: single center experience. J Endourol. 2009; 23: 913-6. 
Submitted for publication:

July 25, 2011

Accepted after revision:

August 23, 2011
Correspondence address:

Dr. Rahul Gupta

3D/D 2nd Extension Surgery

Government Medical College Jammu Green Belt Park,

Gandhinagar

Jammu, Jammu and Kashmir, 180004, India

Fax: + 91979 622-1166

E-mail: rajaguptadr@rediffmail.com 